\title{
Digitale Lernszenarien in der Hochschullehre Bedeutung und Funktion aus Sicht von Studierenden
}

\author{
Claudia Schmidt, Maren Utz, Volker Sänger
}

\section{Zusammenfassung des Beitrags}

Bedingt durch die Coronapandemie wurde in den Informatikkursen Software Engineering und Computernetze an der Hochschule Offenburg ein Lernsetting entwickelt, das mehrere digitale Lernszenarien (Online-Sessions, Lernvideos, Wikis, Quiz, Foren und die selbst entwickelte Lernplattform MILearning) integriert. Im Wintersemester 2020/2021 fand eine Evaluierung statt, um den Einsatz der unterschiedlichen digitalen Lernszenarien in der aktuellen Situation zu bewerten und um zu entscheiden, welche Lernszenarien sinnvoll für einen Einsatz nach der Pandemie sind. Aus dem Blickwinkel des Didaktischen Designs spielen dabei die Eignung der Szenarien für die Wissensvermittlung, die Aktivierung der Studierenden und die Betreuung bei Fragen und Problemen eine wichtige Rolle. Die Ergebnisse zeigen, dass Studierende das Lernsetting intensiv nutzen und die angebotenen digitalen Lernszenarien lernförderlich kombinieren.

Schlüsselbegriffe: Digitale Lernszenarien • Hochschullebre • Didaktisches Design • Studierendenbefragung $\bullet$ Informatik

\section{Einleitung}

Digitale Lernszenarien werden an Hochschulen in unterschiedlichen Gebieten seit Jahren eingesetzt (vgl. Wannemacher et al. 2016). Erzwungen durch die Coronapandemie wurde mit Beginn des Sommersemesters 2020 praktisch die komplette Hochschullehre auf digitale Formate umgestellt. Nach nunmehr drei Semestern, die unter Pandemiebedingungen, also nahezu ausschließlich online, stattgefunden haben, fragen sich viele Lehrende und ganze Hochschulen, welche Bedeutung die pandemiebedingte Umstellung auf digitale Lernszenarien für Studierende hat. Dieser Fragestellung wird anhand von Daten aus Informatikkursen an der Hochschule Offenburg auf den Grund gegangen. Die verwendeten Daten basieren auf einer Befragung und anonymisierten Nutzeraktivitäten aus dem Wintersemester 2020/2021 in den Bachelor-Kursen Software Engineering und Computernetze. Bereits an dieser Stelle sei gesagt, dass digitale Lernszenarien ganz prinzipiell von den Studierenden sehr gut akzeptiert wurden. Das größte Manko der digitalen Semester war - nicht überraschend - das weitgehend entfallene soziale Miteinander im Studium. 


\section{Hybride Lernarrangements an Hochschulen}

Lernsettings an Hochschulen umfassen seit vielen Jahren neben Präsenzveranstaltungen wie Vorlesungen, Seminaren, Laboren oder Übungen eine Vielzahl von digitalen Lernszenarien ${ }^{1}$ (z.B. Web-Based-Trainings, Lernvideos oder interaktive Online-Übungen). Es handelt sich um sogenannte bybride Lernarrangements oder Blended Learning Settings, die unterschiedliche Lernszenarien miteinander kombinieren (vgl. Witt/Czerwionka 2013). Dabei liegen die Vorteile von Präsenzveranstaltungen in der persönlichen Kommunikation und dem sozialen Miteinander der Lernenden (vgl. Arnold et al. 2018).

Digitale Lernszenarien unterstützen Lernprozesse durch die zeitliche und räumliche Unabhängigkeit, ein individuelles Lerntempo (vgl. Lackner/Kopp 2014; Ulrich 2016) sowie durch mediale und interaktive Visualisierungen von Lerninhalten (vgl. Arnold et al. 2018). Eine besondere Bedeutung kommt ihnen in Phasen des Selbststudiums zu, in denen die Lernenden individuell und selbstgesteuert neues Wissen erarbeiten oder bereits erlerntes Wissen einüben. Durch eine anschauliche Visualisierung über Grafiken, Videos oder interaktive Animationen kombiniert mit direktem Feedback kann eine intensive Auseinandersetzung mit den Lerninhalten in problembasierten Szenarien unterstützt werden (vgl. Kerres 2018).

Aus dem Blickwinkel des Didaktischen Designs spielen bei der Gestaltung eines Lernangebotes drei Komponenten eine Rolle (vgl. Reinmann 2015). Die Wissensvermittlung beschäftigt sich mit der Auswahl, der Strukturierung und der Aufbereitung von Lerninhalten. Dabei ist die Wissensvermittlung ein darbietender Prozess, der durch eine Aktivierungskomponente ergänzt wird, damit sich Lernende aktiv mit den Lerninhalten auseinandersetzen. Diese aktive Auseinandersetzung kann über Übungen, Projekte oder Aufgaben erfolgen und hat eine Wissenseinübung, eine Wissenserschließung, eine Wissenstransformation oder die Wissenserschaffung zum Ziel. Die dritte Komponente des Didaktischen Designs beschäftigt sich mit der Betreuung und damit mit sozialen Aspekten des Lernens. Betreuung umfasst die Unterstützung bei Problemen und Fragen, Feedback und die Förderung eines lernförderlichen Klimas. Die Betreuung kann in digitalen Lernszenarien über synchrone oder asynchrone Werkzeuge umgesetzt werden, wie beispielsweise virtuelle Klassenräume durch Audio- und Videosessions, Foren und Wikis (vgl. Scholl/Baldus 2016). Ausgenommen sind Phasen des Selbststudiums, in denen keine Betreuung im eigentlichen Sinne stattfindet, aber ein programmiertes Feedback zum Einsatz kommen kann (vgl. Reinmann 2015).

\section{Informatikkurse an der Hochschule Offenburg}

Präsenzveranstaltungen im Bereich der Informatik an der Hochschule Offenburg werden seit mehr als zehn Jahren mit digitalen Lernszenarien angereichert. Auf der Lernplattform Moodle werden vielfältige Informationen als Dokumente und Links bereitgestellt, z.B. die Folien der Vorlesung, Links zu verwendeten Artikeln aus der Literatur oder nützliche Videos. Foren

\footnotetext{
${ }^{1}$ Digitale Lernszenarien werden in diesem Beitrag als digitalisierte Lernelemente und -formate verstanden (vgl. Wannemacher et al. 2016), die unter didaktischen Gesichtspunkten aufbereitet sind.
} 
werden für Online-Diskussionen genutzt, besonders erfolgreich wird im Rahmen der Klausurvorbereitung ein Austauschforum für Diskussionen zu relevanten Klausurthemen bereitgestellt und moderiert.

Darüber hinaus steht in den Kursen Software Engineering, Computernetze und Datenbanken die webbasierte Lernplattform MI-Learning bereit, die neben dem Inhalt der Vorlesungen auch interaktive Übungen und Quiz integriert (vgl. Schmidt/Sänger 2011; MI-Learning o.J.).

Pandemiebedingt wurden ab März 2020 die etablierten digitalen Lernszenarien ausgeweitet (siehe Abbildung 1). Statt Vorlesungen vor Ort wurden von den Dozierenden Lernvideos aufgenommen und im Moodle-Kurs zur Verfügung gestellt. Übungen aus den Vorlesungen zur Vertiefung der Lerninhalte, Zusammenfassungen zum Lernen und Brainstormingphasen wurden als kollaborative Aufgaben in ein Moodle-Wiki verlagert. Weitere Übungen wurden als Moodle-Quiz implementiert und mit Gamification-Elementen angereichert. In Moodle-Foren konnten sich die Studierenden mit Fragen und Diskussionspunkten zu Wort melden; die fehlende persönliche Kommunikation sollte dadurch kompensiert werden. Die genannten asynchronen Lernszenarien wurden durch wöchentliche synchrone Online-Sessions nach Stundenplan ergänzt, in denen wichtige Inhalte wiederholt und nochmals eine Plattform für Fragen der Studierenden angeboten wurde. Laborveranstaltungen, in denen die Studierenden praktische Übungen durchführen, wurden ebenfalls als Online-Session durchgeführt, wobei die notwendige Software auf Servern der Hochschule bereitgestellt wurde.

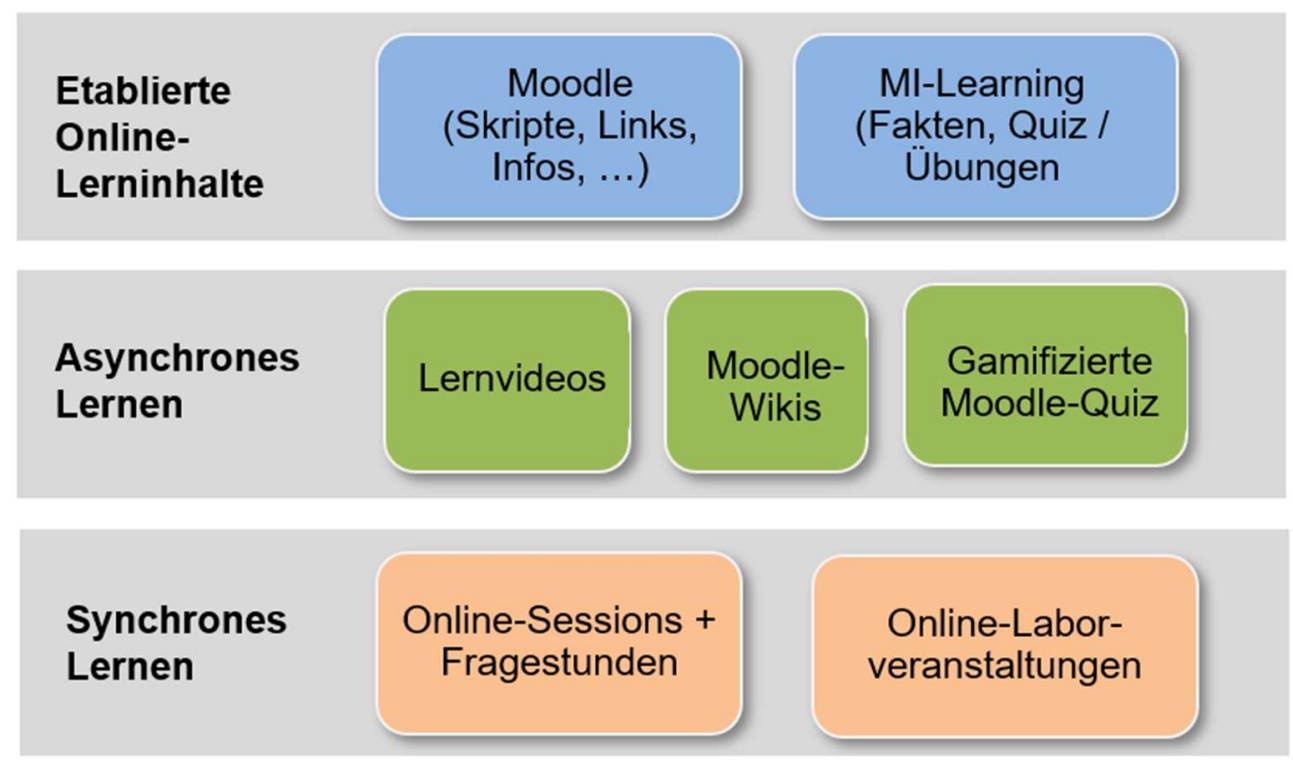

Abbildung 1: Digitales Lernsetting in der Zeit vom Sommersemester 2020 bis Sommersemester 2021

Wie deutlich erkennbar ist, wurde die Lernplattform Moodle als zentraler Anlaufpunkt für das Lernen in der Pandemie weiter ausgebaut. Die Studierenden finden sämtliche Informationen direkt in Moodle oder gelangen über Links auf die externen Server. 
Das beschriebene Lernsetting ist seit dem Coronasemester Winter 2020/2021 im Einsatz ${ }^{2}$ und stellt ein hybrides Lernarrangement dar, bei dem die Wissensvermittlung primär über die erstellten Lernvideos stattfinden soll. Alternativ ist das Nachlesen der Lerninhalte auf der Lernplattform MI-Learning möglich. Die Aktivierung der Studierenden basiert auf den einfachen Aufgaben im Wiki, den vielfältigen und interaktiven Übungen auf der Lernplattform MILearning und den Moodle-Quiz. MI-Learning und die Moodle-Quiz sind zum Selbststudium geeignet und zeichnen sich durch ein direktes Feedback aus. Die Aufgaben im Wiki sind eine Form des kollaborativen Lernens, bei der Studierende und Dozierende gemeinsam zur Lösung beitragen. Schließlich runden die wöchentlichen Online-Sessions das Lernarrangement ab und repräsentieren die Betreuungskomponente zur Diskussion von offenen Aspekten und zur Klärung von Fragen. Alle beschriebenen Lernszenarien sind als Angebote konzipiert und können von den Studierenden auf einer freiwilligen Basis zum Lernen genutzt werden. Einzige Ausnahme stellen ungefähr die Hälfte der Quiz in der Laborveranstaltung Computernetze dar, die verpflichtend absolviert werden müssen.

\section{Evaluation im Wintersemester 2020/2021}

Das beschriebene Lernsetting wurde basierend auf den anonymisierten Zugriffszahlen der Lernplattform Moodle und über eine direkte Befragung der Lernenden am Ende des Wintersemesters 2020/2021 in den beiden Bachelor-Kursen Software-Engineering und Computernetze untersucht. Ziel der Evaluation war es herauszufinden, welche Bedeutung und Funktion die eingesetzten digitalen Lernszenarien für die Studierenden hatten. In Tabelle 1 werden die Zugriffszahlen der verschiedenen Lernszenarien in den beiden betrachteten Kursen in der Zeit vom 1.10.2020 bis 3.3.2021 aufgeführt. Die Werte wurden anonym ermittelt und können den einzelnen Studierenden nicht zugeordnet werden. Bei allen genutzten Lernszenarien, außer MI-Learning, ist trotzdem gesichert, dass sie nur von Lernenden der beiden Kurse genutzt wurden, da ein Zugriff ausschließlich über die Moodle-Kurse möglich ist.

Die Zugriffszahlen in Tabelle 1 zeigen deutlich, dass alle Lernszenarien intensiv genutzt wurden, nur das Klausurforum in Software Engineering fällt erkennbar ab. Beispielhaft seien die Zahlen in der ersten Zeile für das Fach Computernetze wie folgt beschrieben:

1. Die Lernvideos im Kurs Computernetze wurden insgesamt $4.466 \mathrm{Mal}$ aufgerufen. (Ob die Videos damit komplett abgespielt worden sind, lässt sich nicht feststellen.)

2. Dividiert durch die Anzahl vorhandener Lernvideos ergibt sich eine durchschnittliche Anzahl der Zugriffe pro Video von 159,5.

3. Im Durchschnitt wurde ein Lernvideo von 68,8 Nutzenden aufgerufen.

4. Aus 2. und 3. lässt sich ableiten, dass die Lernvideos von den Nutzenden vielfach aufgerufen wurden - im Durchschnitt mehr als zweimal pro Nutzenden.

\footnotetext{
${ }^{2}$ Im ersten Coronasemester (Sommersemester 2020) wurden die Lernszenarien sukzessive entwickelt und standen daher erst im Wintersemester 2020/2021 komplett zur Verfügung.
} 
In welcher Funktion im Lernprozess die einzelnen Lernszenarien von den Studierenden genutzt wurden, ist aus den Zahlen der Tabelle 1 aber nicht ersichtlich. Im Hinblick auf das Didaktische Design, bleibt zu klären welche Lernszenarien von den Studierenden gewählt wurden, um sich neues Wissen anzueignen, um das bestehende Wissen zu erproben oder zu vertiefen und welche Lernszenarien als hilfreich bei Fragen und Problemen erachtet werden (z.B. während der Prüfungsvorbereitung).

\begin{tabular}{|c|c|c|}
\hline & $\begin{array}{l}\text { Kurs Software- } \\
\text { Engineering }\end{array}$ & Kurs Computernetze \\
\hline $\begin{array}{l}\text { Lernvideos } \\
\text { - Anzahl Videos } \\
\text { - Zugriffe } \\
\text { - durchschnittl. Anz. Zugriffe pro Video } \\
\text { - durchschnittl. Zahl der Nutzenden }\end{array}$ & $\begin{array}{l}19 \\
1.581 \\
83,2 \\
41,1\end{array}$ & $\begin{array}{l}28 \\
4.466 \\
159,5 \\
68,8\end{array}$ \\
\hline $\begin{array}{l}\text { Moodle-Wiki } \\
\text { - Zugriffe } \\
\text { - durchschnittl. Anz. Zugriffe pro Wiki-Seite } \\
\text { - durchschnittl. Zahl der Nutzenden }\end{array}$ & \begin{tabular}{|l}
1.422 \\
64,6 \\
30,8
\end{tabular} & $\begin{array}{l}2.450 \\
90,7 \\
40,1\end{array}$ \\
\hline $\begin{array}{l}\text { Moodle-Quiz } \\
\text { - Anzahl Quizseiten } \\
\text { - Zugriffe } \\
\text { - durchschnittl. Anz. Zugriffe pro Seite } \\
\text { - durchschnittl. Zahl der Nutzenden }\end{array}$ & $\begin{array}{l}75 \\
29.282 \\
390,4 \\
40,2\end{array}$ & $\begin{array}{l}39 \\
15.975 \\
409,6 \\
61,2\end{array}$ \\
\hline $\begin{array}{l}\text { MI-Learning Webseite } \\
\text { - Seitenzugriffe } \\
\text { - } \quad \text { Zahl der Besuche } \\
\text { - Seitenzugriffe über Link in Moodle }\end{array}$ & $\begin{array}{l}\text { Nicht bekannt }{ }^{3} \\
\text { Nicht bekannt } \\
215\end{array}$ & \begin{tabular}{|l}
23.938 \\
3.237 \\
819
\end{tabular} \\
\hline $\begin{array}{l}\text { Klausurforum }{ }^{4} \\
\text { - } \quad \text { Zugriffe } \\
\text { - } \quad \text { Zahl der Nutzenden }\end{array}$ & $\begin{array}{l}716 \\
49\end{array}$ & $\begin{array}{l}5.251 \\
75\end{array}$ \\
\hline
\end{tabular}

Tabelle 1: Nutzung der angebotenen Lernszenarien durch Studierende

Um diese Aspekte zu untersuchen, wurde im Rahmen der Evaluierung im Wintersemester 2020/2021 eine Online-Befragung in den Bachelor-Kursen Computernetze und Software Engineering durchgeführt, an der 52 Studierende teilnahmen (vgl. Utz 2021). Die Studierenden kamen aus unterschiedlichen Semestern und aus zwei verschiedenen Bachelor-Studiengängen, wobei der Schwerpunkt auf den niedrigen Semestern lag. Die meisten Studierenden haben im Rahmen der wöchentlichen Online-Sessions an der Befragung teilgenommen, weshalb dabei die in den Kursen aktiven Personen für die Bewertung der Lernszenarien erreicht wurden.

\footnotetext{
${ }^{3}$ Die Zugriffszahlen zum Kurs Software Engineering stehen wegen eines Problems in der Tracking-Software nicht zur Verfügung.

${ }^{4}$ Für die Betrachtung der Foren wurden die Klausurforen ausgewählt, weil diese am stärksten genutzt wurden.
} 
Die Grundgesamtheit der Studierenden der beiden Kurse kann nicht durch die Teilnehmenden in den Moodle-Kursen angegeben werden, da immer Studierende eingetragen sind, die im aktuellen Semester nicht aktiv teilnehmen. Am besten kann man die Grundgesamtheit durch die Zahl der Klausurteilnehmenden abschätzen, was einer Rücklaufquote von ca. 50 Prozent entspricht. Den Kurs Software Engineering belegten Studierende aus dem dritten Semester, wohingegen die Studierenden im Kurs Computernetze entweder aus dem ersten Semester oder aus dem vierten oder höheren Semestern kamen. Es bleibt zu erwähnen, dass die Erstsemester noch kein normales Präsenzstudium erleben konnten (sofern sie nicht schon ein anderes Fach studiert hatten), während alle anderen zumindest ein Präsenzsemester studierten. Allerdings starteten auch die Erstsemester in der ersten Phase des Wintersemesters 2020/2021 teilweise mit Präsenzvorlesungen.

Untersuchungsgegenstand der Evaluierung war das in beiden Kursen genutzte und oben beschriebene Lernsetting, welches um die vor Corona zentrale Präsenzveranstaltung in Form einer Vorlesung ergänzt wurde. Die Bewertung und Bedeutung der Lernszenarien zu den Komponenten Wissensvermittlung, Wissensvertiefung/Wissenseinübung sowie zur Betreuung und dem Austausch bei Fragen und Problemen erfolgte entweder über eine Likert-Skala mit fünf Bewertungspunkten oder über vorgegebene Antwortalternativen (vgl. Utz 2021).

Methodisch ist bei der Ergebnisentwicklung der Befragung zu beachten, dass Studierende in höheren Semestern einige der Lernszenarien aus dem Lernsetting bereits kennen und über Erfahrung im Umgang und in der Arbeit mit den Lernszenarien verfügen. Im Gegensatz dazu haben die niedrigen Semester weniger Erfahrung, weshalb die Ergebnisse und Bewertungen der Lernszenarien unterschiedlich ausfallen können. Zusätzlich können die Bewertungen von den positiven oder negativen Erfahrungen der höheren Semester beeinflusst sein.

\section{Ergebnisse der Evaluation}

Im Nachfolgenden werden ausgewählte Ergebnisse der Evaluierung zur Bedeutung von Lernszenarien in der Hochschullehre aus Sicht der Studierenden vorgestellt. Die Bedeutung der eingesetzten Lernszenarien für die Studierenden im individuellen Lernprozess wurde über folgende Fragestellung ermittelt: Welches Lernszenario hat bei den folgenden Komponenten die größte Bedeutung? Für jede der beschriebenen Komponenten des Didaktischen Designs (Wissensvermittlung, Wissensvertiefung/Wissenseinübung, Betreuung und Austausch) wurde separat erfragt, welches der eingesetzten Lernszenarien am Bedeutendsten für die Studierenden sei. Dabei wurden absolute Werte ermittelt, da die Studierenden immer nur ein Lernszenario auswählen konnten. Den Studierenden standen bei jeder Abfrage die sieben eingesetzten Lernszenarien in den Kursen als Auswahl bzw. Antwortalternativen zur Verfügung.

Das Diagramm in Abbildung 2 fasst die Ergebnisse auf die Frage „Welches Lernszenario hat die größte Bedeutung?” zusammen und zeigt zumeist eindeutige Ergebnisse für einzelne Lernszenarien, die in einer Komponente für die Studierenden von zentraler Bedeutung sind. 
Bei der Wissensvermittlung sind zwei eindeutige Ausprägungen zu erkennen. 42 Prozent der Studierenden bewerten das Lernvideo als zentrales Lernszenario, um sich neues Wissen anzueignen. Daneben wird die Präsenzvorlesung mit 31 Prozent der Stimmen als wichtig für die Wissensaneignung angesehen.

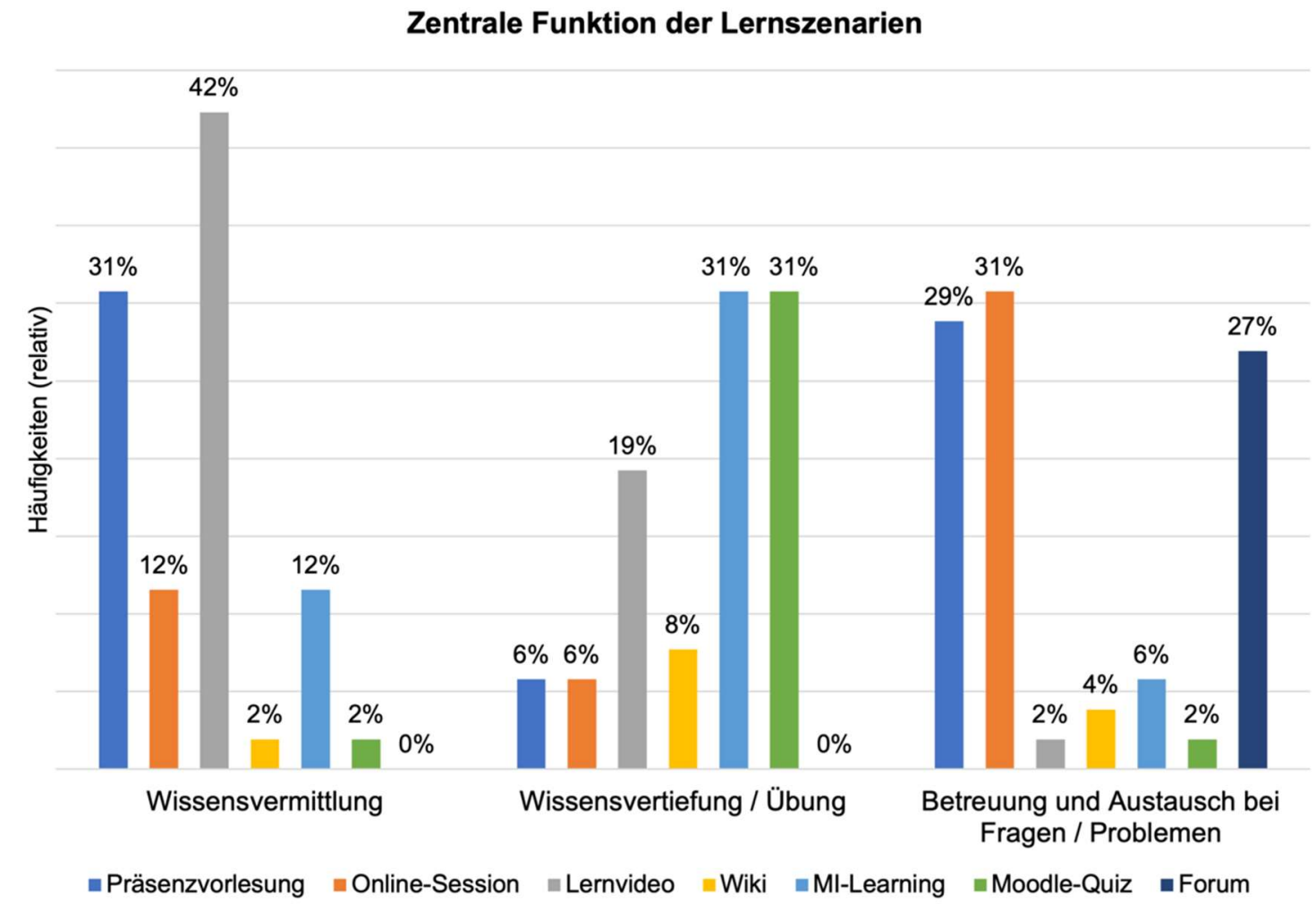

Abbildung 2: Ergebnisdiagramm zentrale Komponenten der Lernszenarien

Für die Vertiefung und Einübung von Wissen sehen die Studierenden vorwiegend die interaktive Plattform MI-Learning und die Moodle-Quiz als zentrale Lernszenarien an (beide 31 \%). Als Option mit $19 \%$ Zustimmung werden die Lernvideos genutzt, um das erlernte Wissen zu wiederholen oder zu vertiefen.

Zur Unterstützung bei Fragen und Problemen oder zum Austausch wird von $31 \%$ der Studierenden die Online-Session als bedeutend angesehen, um mit anderen Lernenden oder Lehrenden in Kontakt zu treten und den Austausch zu suchen. Ähnlich bedeutsam für die Betreuung und den Austausch bei Fragen oder Problemen werden die Präsenzvorlesung (29\%) und Foren (27\%) eingeordnet. Abschließend lässt sich zu diesem Ergebnisteil zusammenfassen, dass alle Lernszenarien außer dem Wiki eine bedeutende Rolle in einer der drei Komponenten einnehmen.

Im zweiten Bereich der Befragung wurden die drei genannten Komponenten des Didaktischen Designs detailliert betrachtet, wodurch die Studierenden alle verfügbaren Lernszenarien im 
Hinblick auf die vorgegebenen Komponenten (Wissensvermittlung, Wissensvertiefung/Wissenseinübung, Betreuung und Austausch) anhand einer bipolaren Likert-Skala (trifft überhaupt nicht $z u$, trifft nicht $z u$, weder noch, trifft zu, trifft voll und ganz zu) bewerten sollten. Diese Ergebnisse ermöglichen eine tiefere Betrachtung, welche Lernszenarien die Studierenden im Lernprozess nutzen und wie sie diese einschätzen. Zudem liefern die Antworten Erkenntnisse zum Vergleich alternativer Lernszenarien, bieten einen Vergleich zwischen der studentischen Bewertung und den tatsächlichen Zugriffszahlen aus den Moodle-Kursen und sollen genutzt werden, um den Einsatz digitaler Lernszenarien für die Zeit nach der Coronapandemie zu evaluieren.

Abbildung 3 repräsentiert eine Zusammenschau der Ergebnisse, wobei ausschließlich die Zustimmung fokussiert ist. Die einzelnen Werte ergeben sich durch die Addition der Prozentzahlen beider möglichen positiven Bewertungspunkte. Im Hinblick auf die Wissensvermittlung gaben beispielsweise 82 \% der befragten Studierenden an, dass sie der Aussage „In einer Präsenzveranstaltung eigne ich mir neues Wissen an" mit trifft zu oder trifft voll und ganz zu bewertet haben.

\section{Zustimmungswerte Lernszenarien}

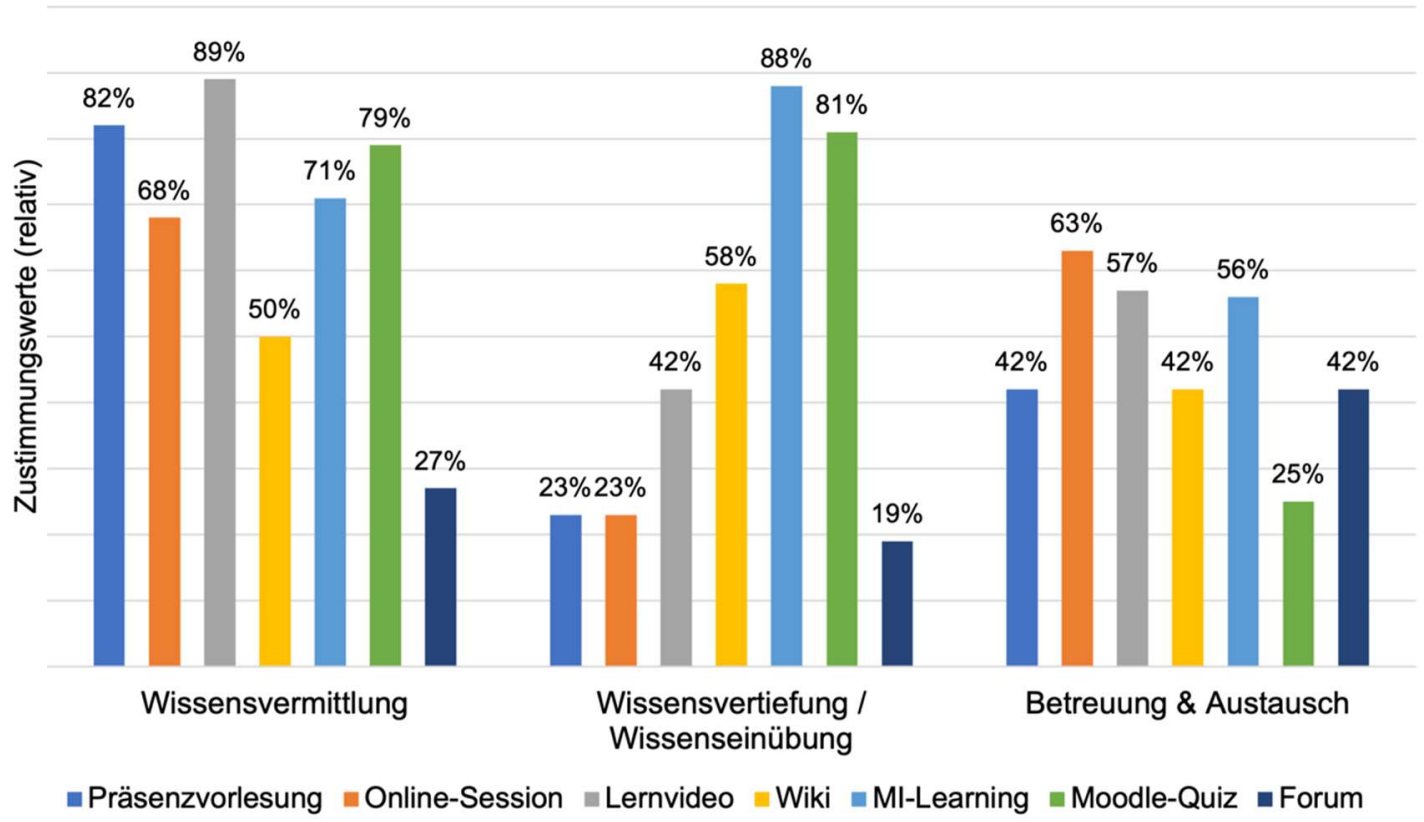

Abbildung 3: Diagramm der Zustimmungswerte (in Prozent) der Lernszenarien im Hinblick auf die Komponenten des Didaktischen Designs

Wissensvermittlung: In dieser Komponente sind die verfügbaren Lernszenarien mit der Aussage „Mit diesem Lernszenario eigne ich mir neues Wissen an” zu bewerten. Die hohen Zustimmungswerte zu den Lernvideos im Hinblick auf die Wissensvermittlung (89\%, davon $15 \%$ trifft voll und ganz zu) sind sicher zum Teil dem onlinebasierten Lernsetting bedingt durch die Coronapandemie geschuldet. Aber darüber hinaus spielen Videos beim Lernen für 
die jüngere Generation eine große Rolle (vgl. Rat für Kulturelle Bildung 2019). Obwohl Lernvideos nur Inhalte vermitteln und keine Möglichkeit für Rückfragen bieten, überwiegen auf der positiven Seite die ständige Verfügbarkeit und die Anpassungen an die Lernenden durch ein selbstgewähltes Tempo und die Wiederholungen von Inhalten (vgl. Wannemacher et al. 2016).

Neben den Lernvideos sehen die Studierenden die Präsenzveranstaltung als bestbewertete Möglichkeit (82\%, davon $31 \%$ trifft voll und ganz zu), um sich neues Wissen anzueignen. Interessant ist, dass MI-Learning und die Moodle-Quiz ebenfalls sehr hohe Zustimmung (71 und 79\%) erfahren, obwohl speziell die Quiz für die Wissensvertiefung und Wissenseinübung entwickelt wurden. Hier zeigt sich, dass die Studierenden neben der expositorischen Darbietung von Inhalten, das aktive Lernen an Beispielen oder Problemen wählen. Dieses Lernsetting lässt Merkmale von problembasiertem Lernen erkennen und ist nach Kerres (vgl. 2018) mit mehr Spaß beim Lernen verbunden, jedoch wird der Einfluss auf den Lernerfolg in Studien unterschiedlich bewertet und weist damit auf weitere zu untersuchende Aspekte hin. Auch die Online-Session und das Wiki leisten einen positiven Beitrag bei der Wissensvermittlung. Alleine das Forum fällt bei dieser Komponente ab.

Die Ergebnisse zeigen, dass Studierende im Hinblick auf die Wissensvermittlung nicht auf einzelne Lernszenarien fixiert sind, sondern mehrere der angebotenen Lernszenarien nutzen und die Vorteile von synchronem/asynchronem und expositorischem/aktivem Lernen kombinieren.

Wissensvertiefung und Wissenseinübung: Hierbei werden die Lernszenarien mit der Aussage „Mit diesem Lernszenario übe ich das erlernte Wissen” bewertet. Bei der Wissensvertiefung und -einübung spielen für die Studierenden die interaktive Lernplattform MI-Learning (46\% trifft voll und ganz zu) und die Moodle-Quiz (35\% trifft voll und ganz zu) eine extrem große Rolle (beide haben Zustimmungswerte über $80 \%$ - siehe Abbildung 3). Beide Szenarien zeichnen sich dadurch aus, dass das Wissen an Beispielen erprobt wird und direktes, programmiertes Feedback gegeben wird. Die Rückmeldungen, die den Lernenden direkt nach dem Bearbeiten einer Aufgabe angeboten werden, zeigen, ob die Aufgabe korrekt gelöst wurde und unterstützen den zukünftigen Lernprozess, da die erhaltene Information direkt bei der Lösung der nächsten Aufgabe genutzt werden kann (vgl. Narciss 2006). Tabelle 1 zeigt bei den MoodleQuiz und bei MI-Learning über sehr hohe Zugriffszahlen, dass Studierende viele Quiz und Übungen mehrfach absolviert haben und speziell in der Zeit vor der Klausur bereits erfolgreich gelöste Quiz nochmals absolviert wurden, um das Wissen aufzufrischen. In MI-Learning existiert außerdem eine Vielzahl von Anwendungen, die sich durch eine hohe Interaktivität auszeichnen. Diese wirkt sich motivierend auf die Lernenden aus und erlaubt ein exploratives Lernen, bei dem Hypothesen aufgestellt und anschließend erprobt werden (vgl. Reinmann 2015).

Das Wiki wird (mit $58 \%$ Zustimmung) als weitere Option von den Studierenden zur Übung und Vertiefung von Wissen bewertet, gefolgt von den Lernvideos (42\%), die online verfügbar sind und jederzeit konsumiert werden können. Beim Wiki konnte beobachtet werden, dass nur eine ausgewählte Gruppe von Studierenden aktiv die Inhalte der Wiki-Seiten verfasste und dabei zwar Inhalte ergänzte, aber keine Fehler korrigierte. Bei Fehlern wurde lediglich eine zweite 
Meinung hinzugefügt. Als Datenbasis zum Lernen wurde das Wiki jedoch intensiv von vielen Studierenden genutzt, wie die Zugriffszahlen in Tabelle 1 zeigen. Die Szenarien Online-Session und Forum sehen die Studierenden weniger zur aktiven Wissensvertiefung oder -einübung.

Betreuung und Austausch bei Fragen und Problemen: Zuletzt wurden die Studierenden aufgefordert, die Aussage „Bei Fragen und Problemen nutze ich dieses Lernszenario “in Verbindung mit den verfügbaren Lernszenarien zu bewerten. Für die Komponente Betreuung und Austausch zur Klärung von Fragen und Problemen bewerten die Studierenden laut den Werten in Abbildung 3 die Online-Session (63\%, davon $23 \%$ trifft voll und ganz zu) am höchsten. Aufgrund der virtuellen, aber persönlichen Kommunikation in einer Online-Session mit Videofunktion erhalten die Lernenden direkte Antworten oder Feedback von Lehrenden. Die niedrigen Zustimmungswerte von $42 \%$ bei der Präsenzvorlesung im Hinblick auf die Betreuung und den Austausch bei Fragen sind sicherlich darauf zurückzuführen, dass kaum Präsenzveranstaltungen stattfanden.

Überraschend sind bei dieser Komponente die relativ hohen Zustimmungswerte von Lernvideos und der Lernplattform MI-Learning (mit 57 und $56 \%$ ), da diese Lernszenarien im Rahmen des Selbststudiums genutzt werden. In diesem Kontext gaben 88 \% der Teilnehmenden bei einer weiteren Frage an, dass sie Fragen und Probleme entweder alleine oder mit anderen Studierenden klären und nur 12 \% bevorzugen dazu das Plenum mit einer Lehrperson (vgl. Utz 2021). Von Vorteil ist beim Selbststudium die direkte Verfügbarkeit der beiden Lernszenarien, mit denen Fragen und Probleme zu dem Zeitpunkt geklärt werden können, in dem sie auftreten. Es muss nicht bis zur nächsten Online-Session gewartet werden.

Ebenfalls überraschend ist die Bewertung der Foren, da diese als Kommunikationstool charakterisiert werden (vgl. Witt/Czerwionka 2013), jedoch nur von $42 \%$ (davon $13 \%$ trifft voll und ganz zu) der Studierenden positiv zur Klärung von Fragen und Problemen bewertet werden. In den Kursen wurden einige Foren zur Diskussion integriert, jedoch wurden diese nur wenig genutzt. Eine Ausnahme stellen die in Tabelle 1 aufgeführten Klausurforen dar, in denen zeitnah zur Klausur Fragen und Probleme gemeinsam zwischen Lernenden und Lehrenden diskutiert wurden. Basierend auf den Ergebnissen der Befragung ist festzustellen, dass die Lernenden gemeinsam mit den Lehrenden, aber auch selbstständig nach Lösungen bei Fragen oder Problemen suchen.

\section{Lessons Learned - Bewertung für die Lehre nach der Coronapandemie}

Die pandemiebedingte Umstellung auf die beschriebenen hybriden Lernarrangements in den Bachelor-Kursen Computernetze und Software-Engineering wurde evaluiert, um Einblicke in die Bedeutung der digitalen Lernszenarien für Studierende zu erhalten. Basierend auf den Ergebnissen steht eine Adaption und Weiterentwicklung des Lernsettings auch für die Zeit nach der Coronapandemie im Fokus.

Die Evaluation zeigt, dass sich die Studierenden bei allen Komponenten des Didaktischen Designs nicht auf ein digitales Lernszenario fokussieren, sondern mehrere Szenarien favorisieren. Dabei spielen vermutlich die unterschiedlichen Eigenschaften wie Synchronizität, Eignung für 
das Selbststudium, Interaktivität oder Feedback eine Rolle. Die Kombination von digitalen Lernszenarien und Präsenzveranstaltungen ist nach unserer Einschätzung lernförderlich, da beide Lernsettings Vorteile bringen, indem sie den Studierenden ein differenziertes und individualisiertes Lernen mit Unterstützung und Begleitung der Lehrenden ermöglichen. Diese Einschätzung findet sich auch bei Arnold et al. (vgl. 2018).

Die hohen Zustimmungswerte für die Lernplattform MI-Learning und die Moodle-Quiz, die von den Studierenden selbstständig und im eigenen Lerntempo bearbeitet werden können, sind eine Bereicherung des Lernsettings, auch für die Zeit nach der Pandemie. Weiter erfahren die pandemiebedingt eingeführten Lernvideos eine hohe Zustimmung bei den Studierenden. Die Lernvideos wurden als Screencasts und unter Zeitdruck erstellt. Hier ist auf Dauer eine Verbesserung durch ein professionelles Vorgehen gepaart mit einer didaktischen Aufbereitung der Lerninhalte für das Medium Lernvideo anzustreben. Aktuelle Überlegungen gehen dahin, ausgewählte Themen, die schwieriger zu verstehen sind, über handwerklich gut gemachte ${ }^{5}$ Lernvideos zu erklären und diese auch in Zukunft zur Verfügung zu stellen. Die Wikis wurden von den Studierenden im Vergleich zu den anderen Lernszenarien nicht so positiv bewertet, obwohl sie intensiv genutzt wurden. Dieses Ergebnis könnte darauf basieren, dass motivationale und soziale Aspekte beim Einsatz von Wikis als Kollaborationstool eine Rolle spielen und daher im Bildungskontext oftmals nicht das Potential von Wikis im Internet erreicht wird (vgl. Kimmerle et al. 2015). Aktuell ist daher geplant, die Aufgaben aus den Wikis nach der Pandemie wieder in die Präsenzveranstaltung zu verlegen und gemeinsam als kollaboratives Lernen dort zu bearbeiten.

Soziale Kontakte spielen beim Lernen eine bedeutende Rolle (vgl. Kerres 2018). Dies bestätigte sich auch in der Befragung, bei der sich die Studierenden im Hinblick auf die Frage „Welches Lernszenario hat die größte Bedeutung für den sozialen Kontakt zu anderen Lernenden?” mit 63 \% für die Präsenzveranstaltung entschieden. Mit nur 27 \% Zustimmung kann eine Online-Session das soziale Lernsetting in Präsenz offenbar nicht ersetzen.

Erwähnenswert ist die Tatsache, dass die Klausurergebnisse in den beiden Coronasemestern, mit dem geschilderten digitalen Lernsetting, vergleichbar zu den Ergebnissen vor der Pandemie waren, teilweise sogar mit besseren Noten. Selbstverständlich können viele unterschiedliche Faktoren zu guten Klausurergebnissen beitragen. Aber zumindest scheint das digitale Lernsetting keinen erkennbar negativen Einfluss zu haben. Dies ist durchaus beachtlich unter den schwierigen Studienbedingungen während der Pandemie.

Allerdings bedeutet die Fortführung der digitalen Lernszenarien zusätzliche Arbeit für die Lehrenden, denn alle Komponenten müssen ständig gepflegt und weiterentwickelt werden, weil die Lerninhalte im Bereich der Informatik einem ständigen Wandel unterworfen sind.

\footnotetext{
${ }^{5}$ Basierend auf einem didaktischen Konzept, einem Drehbuch und einer professionellen Umsetzung (vgl. Ebner/Schön 2017).
} 
Diese Pflege ist eine aufwändige Arbeit und mit der Nutzung sowie Zufriedenheit der Studierenden abzugleichen.

\section{Fazit}

Durch die Coronapandemie wurde kurzfristig die klassische Hochschullehre um digitale Lernszenarien erweitert. Nach dem Ende der Pandemie sollte aber nicht zum Stand der Lehre vor der Pandemie zurückgekehrt werden. Denn die Digitalisierung der Lehre hat einige positive Effekte beschert, die für die Zukunft bewahrt und ausgebaut werden sollten. Dies bedeutet nicht, dass die Präsenzlehre abgeschafft werden soll. Vielmehr soll sie in bestimmten Bereichen sogar intensiviert werden; beispielsweise überall dort, wo der persönliche Kontakt und die persönliche Kommunikation hilfreich sind. In anderen Bereichen dagegen, beispielsweise im Selbststudium, kann auf digitale Szenarien gesetzt werden - etwa auf Videos und Online-Umgebungen, die selbstbestimmt zum individuellen Lernen genutzt werden können.

\section{Literatur}

Arnold, Patricia/Kilian, Lars/Thillosen, Anne/Zimmer, Gerhard (2018): Handbuch E-Learning. Lehren und Lernen mit digitalen Medien. Bielefeld: W. Bertelsmann Verlag.

Ebner, Martin/Schön, Sandra (2017): Lern- und Lehrvideos: Gestaltung, Produktion, Einsatz. In: Wilbers, Karl/Hohenstein, Andreas (Hrsg.): Handbuch E-Learning. Expertenwissen aus Wissenschaft und Praxis - Strategien, Instrumente, Fallstudien, Bd. 71. Stuttgart: utb-Verlag, S. 1-14.

Kerres, Michael (2018): Mediendidaktik: Konzeption und Entwicklung digitaler Lernangebote. Berlin: De Gruyter Oldenbourg. URL: doi.org/10.1515/9783110456837.

Kimmerle, Joachim/Moskaliuk, Johannes/Oeberst, Aileen/Cress, Ulrike (2015): Learning and Collective Knowledge Construction With Social Media. A Process-Oriented Perspective. In: Educational Psychologist, 50 (2), pp. 120-137. URL: 10.1080/00461520.2015.1036273.

Lackner, Elke/Kopp, Michael (2014): Lernen und Lehren im virtuellen Raum: Herausforderungen, Chancen, Möglichkeiten. In: Rummler, Klaus (Hrsg.): Lernräume gestalten - Bildungskontexte vielfältig denken, Münster: Waxmann, S. 174-186. URL: 10.978.38309/31423.

MI-Learning (o.J.): E-Learning der Fakultät Medien und Informationswesen. MI-Learning. URL: mi-learning.hs-offenburg.de (30.08.2021).

Narciss, Susanne (2006): Informatives tutorielles Feedback. Entwicklungs- und Evaluationsprinzipien auf der Basis instruktionspsychologischer Erkenntnisse. Reihe Pädagogische Psychologie und Entwicklungspsychologie, Bd. 56. Münster: Waxmann.

Rat für Kulturelle Bildung (2019): JUGEND/YOUTUBE/ KULTURELLE BILDUNG. HORIZONT 2019. URL: www.rat-kulturelle-bildung.de/fileadmin/user_upload/pdf/Studie_YouTube_Webversion_final.pdf (30.09. 2021)

Reinmann, Gabi (2015): Studientext Didaktisches Design. Hamburg. URL: gabi-reinmann.de/wpcontent/uploads/2013/05/Studientext_DD_Sept2015.pdf(30.08.2021).

Schmidt, Claudia/Sänger, Volker (2011): Fünf Jahre E-Learning in der Informatiklehre - Einsichten und Erkenntnisse. In: Online-Magazin Ludwigsburger Beiträge für Medienpädagogik, Ausgabe 14/2011. URL: doi.org/10.21240/lbzm/14/06.

Scholl, Hartmut/Baldus, Andrea (2016): Training and Qualification: Essentials of New Learning. In: Matthias, Zeuch (Hrsg.): Handbook of Human Resources Management. Berlin/Heidelberg: Springer, S. 293-333. URL: link.springer.com/10.1007/978-3-662-44152-7_28 (30.09.2021). 
Ulrich, Immanuel (2016): Gute Lehre in der Hochschule: Praxistipps zur Planung und Gestaltung von Lehrveranstaltungen. Wiesbaden: Springer. URL: 10.1007/978-3-658-11922-5.

Wannemacher, Klaus/Jungermann, Imke/Scholz, Julia/Tercanli Hacer (2016): Digitale Lernszenarien im Hochschulbereich (Arbeitspapier Nr. 15). HIS-Institut für Hochschulentwicklung (HIS-HE).

Witt, Claudia de/Czerwionka, Thomas (2013): Mediendidaktik. Bielefeld: W. Bertelsmann Verlag. URL: elibrary.utb.de/doi/pdf/10.3278/9783763953165 (30.09.2021).

Utz, Maren (2021). Lernform Blended Learning - Analyse von Lernszenarien in der Hochschullehre (Masterarbeit, Medien und Kommunikation). 


\section{Informationen zu den Autoren}

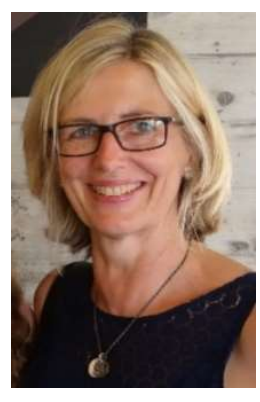

Claudia Schmidt ist Professorin für Computernetze an der Fakultät Medien der Hochschule Offenburg. Ihre Forschungsgebiete sind Next Generation Internet und E-Learning im Bereich der Informatik. Seit mehr als 15 Jahren hat sie mehrere webbasierte E-Learning-Anwendungen für den Einsatz in der Hochschullehre entwickelt. Aktuelle Interessen umfassen die Analyse von Gamification und KI-basierten Chatbots in der Lehre.

c.schmidt@hs-offenburg.de

Maren Utz war Studentin im Masterstudiengang Medien und Kommunikation an der Fakultät Medien der Hochschule Offenburg.

Im Rahmen ihrer Masterarbeit (Wintersemester 2020/2021) zum Thema Blended Learning und dem Einsatz von Lernszenarien in der Hochschullehre untersuchte sie die Lernform und die Funktionen von Lernszenarien aus Sicht von Studierenden. Die Ergebnisse der Untersuchung tragen zur Erarbeitung dieses Beitrags bei.

mutz2@stud.hs-offenburg.de
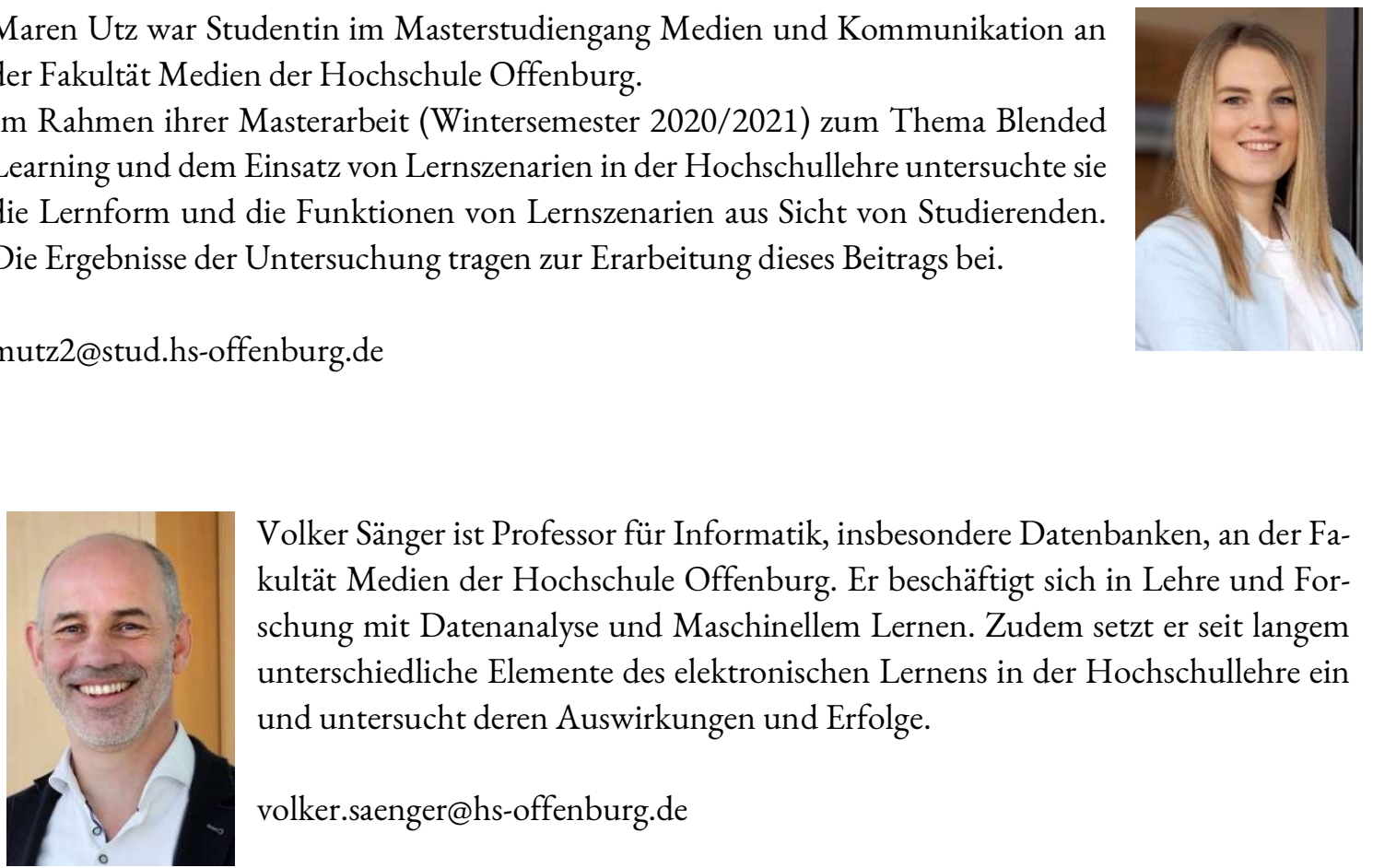

Volker Sänger ist Professor für Informatik, insbesondere Datenbanken, an der Fakultät Medien der Hochschule Offenburg. Er beschäftigt sich in Lehre und Forschung mit Datenanalyse und Maschinellem Lernen. Zudem setzt er seit langem unterschiedliche Elemente des elektronischen Lernens in der Hochschullehre ein und untersucht deren Auswirkungen und Erfolge.

volker.saenger@hs-offenburg.de

\section{Zitationshinweis:}

Schmidt, Claudia/Utz, Maren/Sänger, Volker (2021): Digitale Lernszenarien in der Hochschullehre - Bedeutung und Funktion aus Sicht von Studierenden. In: Online-Magazin Ludwigsburger Beiträge zur Medienpädagogik, Ausgabe 21/2021. URL: medienpaed-ludwigsburg.de/ 